\title{
Conservative Management of Paget Disease of the Breast with Radiotherapy
}

\section{0- and 15-Year Results}

\author{
Jennifer K. Marshall, M.R.c.P. ${ }^{1}$ \\ Kent A. Griffith, M.s. ${ }^{2}$ \\ Bruce G. Haffty, M.D. ${ }^{3}$ \\ Lawrence J. Solin, M.D. ${ }^{4}$ \\ Frank A. Vicini, M.D. ${ }^{5}$ \\ Beryl McCormick, M.D. ${ }^{6}$ \\ David E. Wazer, M.D. ${ }^{7}$ \\ Abram Recht, M.D. ${ }^{8}$ \\ Lori J. Pierce, M.D. ${ }^{1}$ \\ ${ }^{1}$ Department of Radiation Oncology, University of
Michigan School of Medicine, Ann Arbor, Michigan.
${ }^{2}$ Biostatistics Core, University of Michigan Cancer
Center, Ann Arbor, Michigan.
${ }^{3}$ Department of Therapeutic Radiology, Yale Uni-
versity School of Medicine, New Haven, Connect-
icut.
}

${ }^{4}$ Department of Radiation Oncology, University of Pennsylvania Hospital, Philadelphia, Pennsylvania.

${ }^{5}$ Department of Radiation Oncology, William Beaumont Hospital, Royal Oak, Michigan.

${ }^{6}$ Department of Radiation Oncology, Memorial Hospital, Memorial Sloan-Kettering Cancer Center, New York, New York.

${ }^{7}$ Department of Radiation Oncology, Tufts New England Medical Center, Boston, Massachusetts.

${ }^{8}$ Department of Radiation Oncology, Beth Israel Deaconess Medical Center, and Harvard Medical School, Boston, Massachusetts.

Presented at the 44th Annual Meeting of the American Society of Therapeutic Radiology and Oncology, New Orleans, Louisiana, October 6-10, 2002.

Address for reprints: Lori J. Pierce, M.D., Department of Radiation Oncology, University of Michigan School of Medicine, Cancer and Geriatrics Center, 1500 E. Medical Center Drive, Room 4308, Ann Arbor, Ml 48109; Fax: (734) 647-9654; E-mail: ljpierce@umich.edu

Received October 23, 2002; revision received December 30, 2002; accepted January 9, 2002.
BACKGROUND. At 5-year follow-up, patients with Paget disease of the breast who were treated with breast-conserving surgery (BCS) and radiotherapy (RT) had excellent results. The current report provides 10- and 15-year rates of tumor control in the breast, as well as disease-free and overall survival rates following BCS and RT in a cohort of patients with Paget disease presenting without a palpable mass or mammographic density.

METHODS. Through a collaborative review of patients treated with BCS and RT from seven institutions, 38 cases of Paget disease of the breast presenting without a palpable mass or mammographic density were identified. All patients had pathologic confirmation of typical Paget cells at time of diagnosis. Thirtysix of 38 patients had a minimum follow-up greater than 12 months and constitute the study cohort. Ninety-four percent of patients underwent complete or partial excision of the nipple-areola complex and all patients received a median external beam irradiation dose of 50 Gy (range, 45-54 Gy) to the whole breast. Ninety-seven percent of patients also received a boost to the remaining nipple or tumor bed, a median total dose of 61.5 Gy (range, 50.4-70 Gy).

RESULTS. With median follow-up of 113 months (range, 18-257 months), 4 of 36 patients $(11 \%)$ developed a first recurrence of disease in the treated breast only. Two of the four recurrences in the breast were ductal carcinoma in situ (DCIS) only and two were invasive with DCIS. Two additional patients had a recurrence in the breast as a component of first failure. Actuarial local control rates for the breast as the only site of first recurrence were $91 \%$ at 5 years $(95 \%$ confidence interval [CI], 80-100\%) and 87\% (95\% CI, 75-99\%) at both 10 and 15 years. Actuarial local control rates for breast recurrence, as a component of first failure, were $91 \%$ (95\% CI, 80-100\%), 83\% (95\% CI, 69-97\%), and 76\% (95\% CI, $58-94 \%)$ at 5,10 , and 15 years, respectively. No clinical factors were identified as significant predictors for breast recurrence. Five-, 10- and 15-year actuarial rates for survival without disease of $97 \%$ (95\% CI, 90-100\%) and 5-, 10-, and 15 -year actuarial rates of overall survival of $93 \%$ (95\% CI, 84-100\%) at 5 years and $90 \%(95 \% \mathrm{CI}, 78-100 \%)$ at 10 and 15 years were reported.

CONCLUSIONS. These data confirm excellent rates of local control, disease-free survial, and overall survival at 10 and 15 years following BCS and RT for Paget disease of the breast. This study continues to support the recommendation of local excision and definitive breast irradiation as an alternative to mastectomy in the treatment of patients with Paget disease presenting without a palpable mass or mammographic density. Cancer 2003;97:2142-9.

(C) 2003 American Cancer Society.

Dol 10.1002/cncr.11337

KEYWORDS: Paget disease, breast carcinoma, radiotherapy, breast conservation. 
$\mathbf{P}$ aget disease of the breast represents $1-3 \%$ of all breast malignancies ${ }^{1-5}$ and is characterized pathologically by the presence of round intraepidermal cells of the nipple. Although the majority of cases of Paget disease will have an underlying breast malignancy, ${ }^{1-4}$ previous studies of patients treated with mastectomy have identified two distinct clinical presentations with associated histologic findings. ${ }^{1,5}$ Approximately 90\% of patients presenting with a palpable or mammographic mass will have an underlying invasive carcinoma. Conversely, $66-86 \%$ of patients without a clinical mass on physical examination or mammogram will have ductal carcinoma in situ (DCIS) alone. ${ }^{1,5}$ Prognosis is determined primarily by the presence or absence of an invasive component, ${ }^{1,2,5}$ and recommendations for systemic therapy have been suggested accordingly.

The role of breast-conserving surgery (BCS) with radiotherapy (RT) for selected patients with Paget disease, however, has not been defined fully. Multiple randomized trials have found BCS and RT to be an acceptable alternative and often preferable option to mastectomy for patients with early-stage invasive breast carcinoma. Randomized trials also have confirmed the highly successful results of BCS and RT in the treatment of patients with DCIS. ${ }^{6-8}$ However, due to the rarity of Paget disease of the breast, a prospective, randomized trial comparing BCS and RT to mastectomy is unlikely to be feasible. Therefore, several retrospective studies have reviewed limited experience with RT alone or conservative surgery with or without the addition of RT in the treatment of Paget disease, but the analyses are limited by small patient numbers with limited follow-up and/or variation in clinical presentation. ${ }^{2,9-13}$ The current report presents an analysis of a collaborative series from seven institutions comprising patients with Paget disease of the breast presenting with nipple changes in the absence of a palpable mass or mammographic density who were treated with BCS and RT. Rates of local control and disease-free and overall survival at 5 years were reported previously. ${ }^{14}$ We report the results with extended follow-up at 10 and 15 years.

\section{MATERIALS AND METHODS}

All patients diagnosed with Paget disease of the breast without a palpable mass or mammographic density and treated with BCS and RT between 1980 and 2000 at 7 collaborating institutions were identified. Patients were treated with breast conservation based on patient preference. No patients were referred for RT due to contraindications to surgery. Previously reported cases were updated by the originating institutions with respect to date of last follow-up, survival status, patterns of failure and complications, as appropriate. All new cases identified since the previous analysis and treated with BCS and RT were added to the dataset and included in the current analysis. Thirty-eight cases of Paget disease of the breast were identified from the following institutions: 10 from the University of Michigan, 9 from Yale University, 5 each from the University of Pennsylvania and William Beaumont Hospital, 4 from Memorial Sloan-Kettering Cancer Center, 3 from Tufts University, and 2 cases from Beth Israel Deaconess Medical Center. Due to follow-up limited to less than 12 months, two patients were excluded from the current analysis.

Each patient had a nipple biopsy demonstrating typical Paget cells at the time of diagnosis. A central pathology review was not performed. Thirty-three women were diagnosed with American Joint Committee on Cancer TisN0M0, Stage 0 Paget disease of the breast. ${ }^{15}$ Two patients were diagnosed with T1N0M0, Stage I Paget disease, one with microinvasion and one with invasive ductal carcinoma measuring $0.5 \mathrm{~cm}$. One patient had T2N0M0, Stage IIA disease with a mammographically occult invasive ductal and lobular carcinoma measuring $2.7 \mathrm{~cm}$ located directly beneath the nipple-areolar complex.

Surgery consisted of either a complete or partial excision of the nipple-areolar complex. Twenty-five patients $(69 \%)$ underwent a complete excision, 9 patients (25\%) a partial excision, and 2 (6\%) underwent a biopsy alone. Final margins were negative in 20 cases (56\%), positive in $2(6 \%)$, and unknown in $14(39 \%)$. Fourteen patients $(39 \%)$ had an axillary lymph node dissection, with a median of 13 lymph nodes resected (range, 8-24 lymph nodes), and one patient had a sentinel lymph node biopsy (four lymph nodes were removed). All patients received RT using tangential fields to the whole breast. The median dose delivered was 50 Gy (range, 45-54 Gy) with median fraction size of 2 Gy (range, 1.8-2 Gy). An area of increased dose (underwedging) in the nipple-areolar region was identified in seven RT plans, each with a maximum isodose of $106-110 \%$. A boost was delivered to the remaining nipple or tumor bed in $97 \%$ of cases to a median dose of $14 \mathrm{~Gy}$ (range, 9-20 Gy). The median total dose to the tumor bed was $61.5 \mathrm{~Gy}$ (range, 50.4-70 Gy). Of the 35 patients receiving a boost, 29 patients $(81 \%)$ were treated with electrons, 1 patient $(3 \%)$ received both electrons and photons, and 3 patients $(9 \%)$ were treated using photons only. Two women $(6 \%)$ received a boost using a low dose rate interstitial implant. Bolus was used in the photon and/or electron portion of treatment in 10 cases (27\%), bolus was not used in 24 
(67\%) cases, and it is unknown whether bolus was used in 2 cases. The location of the placement of bolus was highly variable between institutions. Bolus was applied to the scar or remaining nipple-areolar complex during whole breast RT in six patients, to the breast and scar boost in two patients, and to the scar boost only in two patients. In addition to breast irradiation, one patient received supraclavicular RT to a dose of 46 Gy and a second patient was treated comprehensively to the supraclavicular, axillary, and internal mammary lymph nodes to a total dose of $50 \mathrm{~Gy}$. Two patients $(6 \%)$ received systemic treatment with tamoxifen.

Patient records were reviewed for the following characteristics: age, menopausal status, presenting symptoms, duration of symptoms, mammographic findings, extent of surgical excision, final margin status, pathologic findings, and dissection of axillary lymph nodes with pathologic results. Actuarial curves for local control, disease-free survival, and overall survival were generated using the Kaplan-Meier method measured from the completion of RT. ${ }^{16}$ A local recurrence as only first recurrence was defined as a recurrence in the breast without preceding or simultaneous regional or distant disease. Local recurrence as a component of first recurrence included all patients who developed a breast recurrence either as an isolated recurrence or concurrent with regional and/or distant recurrence. Time to local recurrence was defined as the time between completion of RT and the date of recurrence. Patients were scored as having no evidence of disease (NED) at last observation if they had been continuously free of disease from the completion of RT to the last follow-up visit or had developed local and/or regional recurrence that was successfully salvaged at the last observation period. For the calculation of cause-specific survival, only deaths secondary to breast carcinoma were scored as events.

Paget cases identified since the previous analysis were added to the existing cases, and univariate analyses were performed using the Fisher exact test to evaluate the association between clinical characteristics and local control.

\section{RESULTS}

\section{Clinical and Pathologic Findings}

Thirty-six patients with a follow-up greater than 12 months were identified. Median age at diagnosis was 51 years (range, 33-79 years). Patient characteristics and mammographic and pathologic findings are summarized in Table 1. All patients were symptomatic at presentation with a median duration of symptoms of 9 months (range, 1-36 months).
TABLE 1

Clinical, Mammographic, and Pathologic Characteristics

\begin{tabular}{ll}
\hline Characteristic & No. of patients (\%) \\
\hline Menopause status & \\
Premenopausal & $14(39)$ \\
Perimenopausal & $1(3)$ \\
Postmenopausal & $20(56)$ \\
Unknown & $1(3)$ \\
Presenting symptoms & \\
Eczematous changes & $21(58)$ \\
Nipple discharge & $12(33)$ \\
Erythema & $10(28)$ \\
Ulceration & $5(14)$ \\
Mammographic findings & \\
Negative for malignancy & $27(77)$ \\
Abnormality detected & \\
Nipple thickening & $8(23)$ \\
Subareolar calcifications only & 3 \\
Breast calcifications elsewhere & 3 \\
Extent of nipple-areolar excision & 3 \\
Complete nipple-areolar complex & \\
Partial nipple-areolar complex & $25(69)$ \\
Incisional biopsy only & $9(25)$ \\
Final margin status & $2(6)$ \\
Negative & \\
Positive & $20(56)$ \\
Unknown & $2(6)$ \\
Underlying malignancy & $14(38)$ \\
DCIS only & $30(83)$ \\
DCIS and invasive carcinoma & $27(75)$ \\
Invasive carcinoma only & $2(6)$ \\
T stage & $1(3)$ \\
Tis & $33(92)$ \\
T1 & $2(6)$ \\
T2 & $1(3)$ \\
\hline &
\end{tabular}

DCIS: ductal carcinoma in situ.

${ }^{a}$ One patient had both nipple thickening and subareolar calcifications.

Pathologic review at the time of diagnosis revealed typical Paget cells of the nipple in all 36 cases. Thirty cases (83\%) had an underlying malignancy. Of the remaining six cases, two patients had no underlying breast parenchyma resected and four had no DCIS or invasion present. Among the 15 patients who underwent axillary lymph node surgery, all lymph nodes were pathologically negative for metastatic disease.

Of six patients with mammographic calcifications (17\%), 4 had a postoperative mammogram. Three showed no residual calcifications and one showed residual subareolar microcalcifications. No further surgery was performed on this patient before RT.

\section{Local Control, Cosmesis, and Complications}

Median follow-up for surviving patients was 113 months (range, 17-257 months). Table 2 shows the 5-, 
TABLE 2

Actuarial Local Control Rates for the Breast as the Only Site of First Recurrence and as a Component of First Recurrence

\begin{tabular}{|c|c|c|}
\hline Year & Control rate (\%) & 95\% Confidence interval \\
\hline \multicolumn{3}{|c|}{ Breast as only site of recurrence } \\
\hline 5 & 91 & $80-100$ \\
\hline 10 & 87 & $75-99$ \\
\hline 15 & 87 & $75-99$ \\
\hline \multicolumn{3}{|c|}{ Component of first recurrence } \\
\hline 5 & 91 & $80-100$ \\
\hline 10 & 83 & $69-97$ \\
\hline 15 & 76 & $58-94$ \\
\hline
\end{tabular}

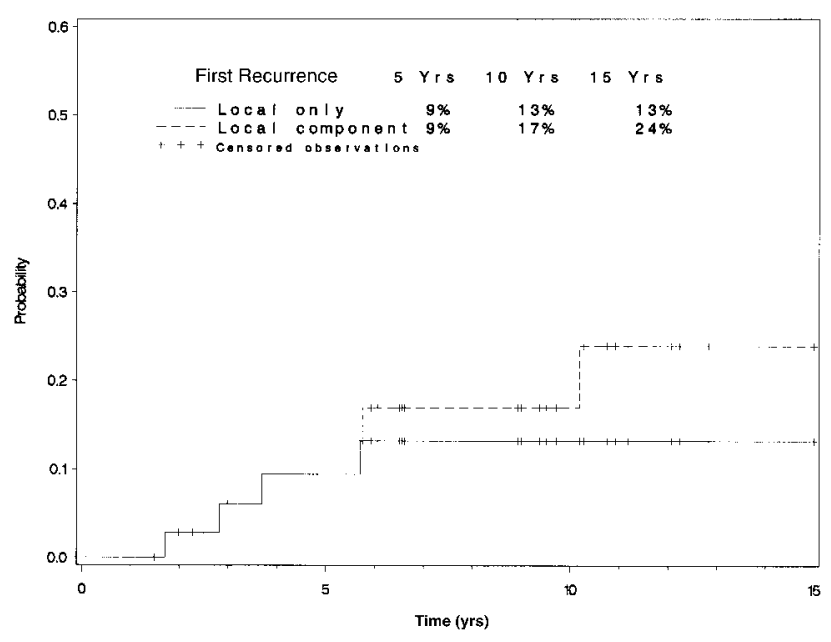

FIGURE 1. Actuarial rates of local recurrence in the breast as the only site of first recurrence and as a component of first failure.

10-, and 15-year actuarial local control rates for the breast as the only site of first recurrence and as a component of first recurrence. Actuarial curves for local recurrence are presented in Figure 1.

Four of 36 patients (11\%) developed local disease recurrence 21-69 months after completion of RT. Surgical margins at primary surgery were negative in two patients and unknown in the other two patients. All patients had undergone complete resection of the nipple-areolar complex at primary surgery, although a postoperative mammogram showed residual subareolar microcalcifications in one patient. All were successfully salvaged by mastectomy. Pathologic findings indicated DCIS only in two patients and DCIS with invasive disease in the remaining two patients.

Two additional patients (6\%) developed an inbreast recurrence simultaneously with either regional or distant recurrence. Pathology revealed invasive and intraductal disease in both cases. Time to local recurrence for these patients was 69 months and 122

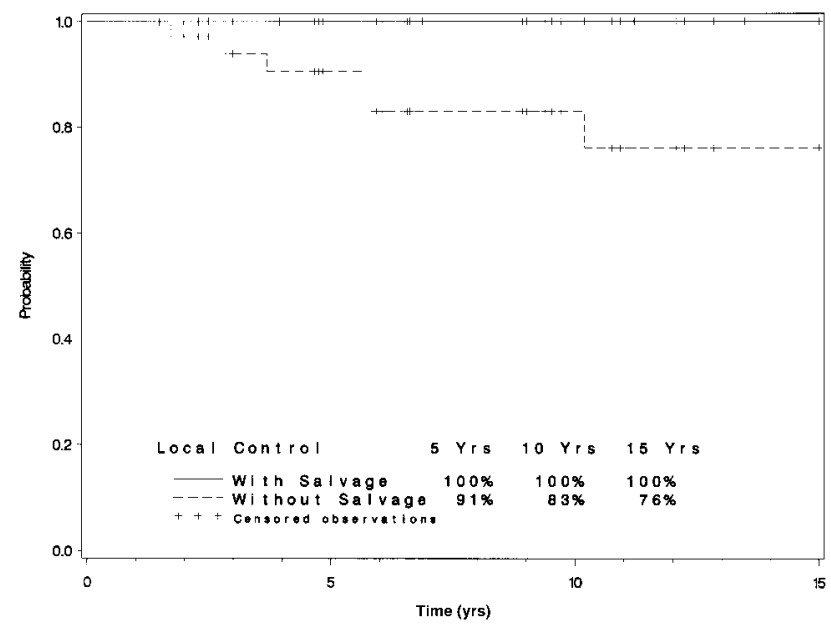

FIGURE 2. Actuarial rates of local control with and without surgical salvage.

months, respectively. Both had a partial nipple-areolar complex excision at primary surgery with final margin status unknown. One woman experienced a simultaneous breast and axillary lymph node recurrence and was treated by mastectomy and axillary lymph node dissection followed by chemotherapy and tamoxifen. She has remained free of disease at last follow-up 11.9 years after disease recurrence. The second patient had a simultaneous recurrence in the breast and contralateral axilla 10.5 years following initial diagnosis. A diagnostic biopsy of the treated breast was performed at that time. It confirmed recurrent disease, but no attempt was made to perform a mastectomy. The patient died with disease 5.5 years following recurrence.

At the time of last follow-up, local disease control was obtained for 35 of 36 patients $(97 \%)$, either by primary treatment (30 of $36 ; 83 \%$ ) or salvage surgery (5 of 5 patients). Actuarial curves of local control with and without surgical salvage are shown in Figure 2. None of the patients with evidence of invasive carcinoma at the time of primary surgery experienced disease recurrence.

Univariate analysis for local failure as a component of first failure was performed for a number of clinical, pathologic, and treatment factors (Table 3). No factors significantly predicted the risk of local disease recurrence.

Cosmesis was assessed using the criteria suggested by Harris et al. ${ }^{17}$ Ten of 31 patients (32\%) were considered by the treating radiation oncologist to have an excellent result, including 4 patients who underwent nipple reconstruction after complete nipple-areolar resection. Eighteen patients $(58 \%)$ were considered to have a good result and 3 patients $(10 \%)$ a fair result. 
TABLE 3

Univariate Analyses for Local Recurrence

\begin{tabular}{|c|c|c|c|}
\hline Clinical factor & $\begin{array}{l}\text { No. of } \\
\text { patients }\end{array}$ & $\begin{array}{l}\text { Local } \\
\text { recurrence (\%) }\end{array}$ & $P^{a}$ \\
\hline \multicolumn{4}{|l|}{ Mammographic findings } \\
\hline Negative & 27 & 19 & $>0.99$ \\
\hline Calcifications & 8 & 13 & \\
\hline Not done & 1 & 0 & \\
\hline \multicolumn{4}{|l|}{ Extent of nipple-areolar excision } \\
\hline Complete nipple-areolar complex & 25 & 16 & 0.65 \\
\hline Partial nipple-areolar complex & 9 & 22 & \\
\hline Not done & 2 & 0 & \\
\hline \multicolumn{4}{|l|}{ Final margin status } \\
\hline Negative & 20 & 10 & $>0.99$ \\
\hline Positive & 2 & 0 & \\
\hline Unknown & 14 & 29 & \\
\hline \multicolumn{4}{|l|}{ Pathologic results } \\
\hline Paget disease only & 6 & 0 & 0.56 \\
\hline $\begin{array}{l}\text { Paget disease with DCIS with/without } \\
\text { invasion }\end{array}$ & 30 & 20 & \\
\hline \multicolumn{4}{|l|}{ Breast dose (Gy) } \\
\hline$<50$ & 13 & 15 & $>0.99$ \\
\hline$\geq 50$ & 23 & 17 & \\
\hline \multicolumn{4}{|l|}{ Dose to tumor bed with boost (Gy) } \\
\hline$<60$ & 4 & 0 & $>0.99$ \\
\hline$\geq 60$ & 32 & 19 & \\
\hline \multicolumn{4}{|l|}{ Use of bolus } \\
\hline Yes & 10 & 30 & 0.33 \\
\hline No & 24 & 13 & \\
\hline Unknown & 2 & 0 & \\
\hline
\end{tabular}

Gy: gray.

${ }^{\text {a }}$ Fisher exact test computed for known clinical factor categories only.

Complications were assessed in 32 of 36 cases, with $91 \%$ (29 patients) having no long-term complications. In addition, one patient developed protracted chest wall pain, one developed chronic breast infection, and one possible radiation dermatitis requiring steroids.

\section{Cause-Specific and Overall Survival Rates}

At last follow-up, 32 of 36 patients (89\%) had NED. Two patients $(6 \%)$ died with disease, and 2 died free of disease. The two patients who died with disease both had a previous breast recurrence. One woman had a simultaneous recurrence in the contralateral axilla and the second patient had a breast cancer recurrence 69 months after completion of RT and underwent a successful salvage mastectomy. However, she developed bone metastases 22 months after breast recurrence and died 5 months thereafter.

Actuarial curves for cause-specific survival and overall survival at 5,10 , and 15 years are provided in Figure 3 . The actuarial rate for breast carcinoma-spe-

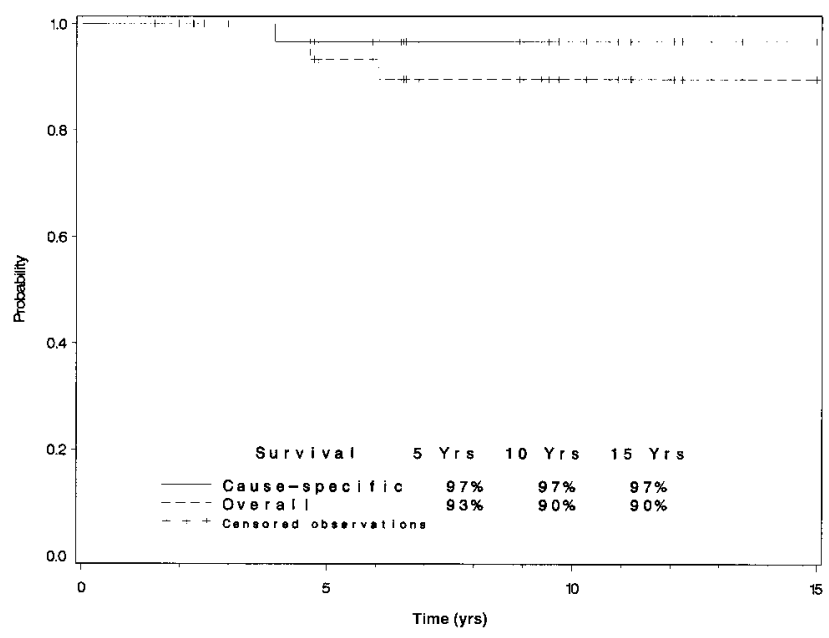

FIGURE 3. Actuarial rates of cause-specific survival and overall survival.

cific survival was $97 \%$ at 5,10 , and 15 years $(95 \%$ confidence interval [CI], 90-100\%). Actuarial rates for overall survival were $93 \%$ (95\% CI, $84-100 \%$ ) at 5 years and $90 \%(95 \%$ CI, $78-100 \%)$ at 10 and 15 years. One patient with disease died at 15.6 years, giving an overall survival beyond 15 years of $75 \%$ (95\% CI, $46-100 \%$ ).

\section{DISCUSSION}

The current study represents an update of the collaborative series reported by Pierce et al. ${ }^{14}$ of patients with Paget disease of the breast presenting without a palpable mass or mammographic density treated with BCS and RT. In the Pierce et al. report, the 5- and 8-year local control rates with the breast as the only site of first recurrence were $91 \%$ and $84 \%$, respectively, the 5- and 8-year disease-free survival rate was $95 \%$, and the 8-year cause-specific overall survival rate was $100 \%$. These results suggested that for selected patients with Paget disease of the breast, BCS and RT was a viable alternative to mastectomy. In the current article, the local control rate of $87 \%$ at 10 and 15 years, the cause-specific survival rate of $97 \%$ at 10 and 15 years, and the overall survival rate of $90 \%$ at 10 and 15 years add further support to the original conclusions.

Small series have shown increased rates of recurrence in the breast following BCS only, with rates of recurrence of $20-60 \%$ at $12-19$ months following surgery. ${ }^{18-20} \mathrm{~A}$ recent report by Polgar et al. ${ }^{21}$ of 33 patients identified in the database of the National Institute of Oncology, Budapest, Hungary, as being treated with cone excision alone represents the largest series ever reported of BCS only. The median age of the patients in their study was 65 years compared with a median age of 51 years in the current study. Ninety- 
one percent of cases in the Polgar et al. study were not associated with a palpable mass, compared with $100 \%$ in the current study. In addition, DCIS was identified in $91 \%$ of cases versus $75 \%$ in the current study. There were no cases of invasive cancer identified in the series from Hungary. With a median of 6.0 years of follow-up (range, 2-14 years), the crude local recurrence rate was $33 \%$ ( 11 of 33 ), with a 5 -year actuarial recurrence rate of $28 \%$. Disease recurrence was invasive in 10 of the 11 patients. Six of these patients developed distant metastases and all have subsequently died of breast carcinoma. These data demonstrate that Paget disease of the breast should not be treated with cone excision only. As stated by the authors, "RT is mandatory after breast-conserving surgery to maintain adequate local control" (p. 1905). ${ }^{21}$ Although direct comparisons cannot be made across series, similar clinicopathologic characteristics between the patients in the surgery-only series and the current study suggest a significant reduction in ipsilateral breast recurrence and improvement in diseasespecific survival in patients treated with breast irradiation.

Other studies have reported the outcome of Paget disease treated with limited surgery and definitive RT. ${ }^{10,12,22}$ In a recent report, the European Organization for Research and Treatment of Cancer (EORTC) ${ }^{22}$ presented their results of a cohort of 61 patients with Paget's disease treated with BCS and RT. With a median follow-up of 6.4 years, the 5-year local recurrence rate was estimated to be $5.2 \%$ compared with the $9 \%$ local recurrence rate in the current study. Again, differences in patient selection and treatment between the two studies preclude direct comparisons between the results. However, some differences should be underscored. The median age of patients in the current study was 51 years compared with 58 years in the EORTC study. The EORTC study included patients with a palpable lesion but excluded patients with histologic evidence of invasive carcinoma, which was present in $8 \%$ of patients in the current study. All patients in the EORTC study received a complete excision of the nipple-areolar complex and margins were required to be histologically tumor free. This compares to a complete nipple-areolar excision in $69 \%$ of patients in the current study with only $56 \%$ of specimens with margins known to be free of microscopic disease. Repeat mammography was performed in all EORTC patients 6 weeks postoperatively before RT whereas only four of the six patients with suspicious calcifications underwent repeat mammography before RT in the current study. Although susbsequent analyses of the EORTC study are required to assess sustained rates of local control, we concur with the need to obtain negative margins of excision and a postbiopsy mammogram negative for residual suspicious microcalcifications before proceeding with breast conservation. These recommendations are in keeping with current practice guidelines in the treatment of both invasive cancer and DCIS.

In almost all patients diagnosed with Paget disease of the breast, underlying carcinoma, either invasive or intraductal, will be identified which will determine prognosis. In patients with Paget disease and no palpable or mammographic mass, the majority will have underlying DCIS. Therefore, axillary lymph nodes are generally pathologically negative as shown in the current report, and treatment should be directed to the breast. For patients with Paget disease presenting with nipple changes alone, the available data support treatment consistent with an assumed underlying DCIS. Conservative management of DCIS using BCS and RT has been highly successful. Two randomized trials have demonstrated significant reduction in the risk of local recurrence including invasive recurrence in patients receiving whole breast RT following lumpectomy. ${ }^{7,8}$ Rates of local control in these series have approximated $90 \%$ at 5 years, similar to the $91 \%$ rate in the current study.

Long-term follow-up data are particularly important for patients with DCIS because DCIS generally has a longer median time to recurrence than invasive carcinoma. In addition, the median time to recurrence following lumpectomy and RT for DCIS is longer than that following lumpectomy alone. ${ }^{7,23}$ One report suggested a median time to recurrence of 8 years or more. ${ }^{24}$ Solin et al. ${ }^{25}$ reported that patients with mammographically detected DCIS treated with BCS and RT had a 15 -year cause-specific survival rate of $98 \%$, with an overall survival rate of $92 \%$. These rates are comparable to those found in the current study, in which the 15 -year cause-specific survival rate was $97 \%$ and the overall survival rate was $90 \%$. The 15 -year rate of local-only recurrence as first recurrence was $14 \%$ compared with $13 \%$ in the current study and the median time to local recurrence was 5 years. This was also a multi-institutional collaborative study with a median follow-up of 9.4 years. With the selection criteria used in the current study, the long-term results of patients with Paget disease of the breast treated with definitive irradiation are highly concordant with results achieved in patients with known DCIS.

Treatment of DCIS by mastectomy, previously the treatment of choice, does not necessarily result in a $100 \%$ cure rate. A metaanalysis of mastectomy patients reported a breast carcinoma-specific mortality 
rate of $1.7 \%$ at 8.6 years of follow-up. ${ }^{26}$ Similarly, the use of mastectomy for Paget disease has also resulted in high rates of local control and survival. ${ }^{2,27,28}$ Some studies, however, report invasive recurrences following surgery. Dixon et al. ${ }^{2}$ reported a $5 \%$ rate of locoregional invasive recurrence in 2 of 37 patients with Paget disease of the breast presenting without a palpable mass following mastectomy. These patients were successfully salvaged however, and remain disease free after 8 years of follow-up. Although the use of mastectomy may result in improved local control compared with breast-conserving therapy, there are obvious cosmetic and psychological consequences. Breast-conserving treatment for Paget disease and DCIS must aim to minimize local recurrence and achieve excellent rates of disease-free and overall survival as demonstrated in the current study. The extent of resection and the adequacy of margins are known factors predictive of local control in the conservative management of DCIS. ${ }^{29,30}$ Although the current study was unable to demonstrate any statistically significant difference in local control for the clinical factors studied, complete excision of all underlying carcinoma should be one of the aims of treatment. For this reason, the authors continue to recommend that the treatment of Paget disease should include excision of the entire nipple-areolar complex, achievement of negative microscopic margins, and removal of all suspicious microcalcifications seen on pre-treatment mammograms before proceeding with definitive RT.

To the best of our knowledge, the current study has the longest median follow-up of any reported series of patients treated with BCS and definitive RT for Paget disease of the breast. Identification of factors associated with local recurrence was not possible in the current study due to the limitations of the small sample of patients and variation in treatment due to the collaborative nature of the study and the time period over which the cases were collected. Other limitations include incomplete information regarding the final pathology margins in $39 \%$ of the patients in this series and the absence of a post-operative mammogram to rule out residual microcalcifications in all patients presenting with suspicious microcalcifications. Despite these limitations, this study demonstrates excellent rates of local control, cause-specific survival, and overall survival at 10 and 15 years. The authors confirm that BCS and RT is an appropriate alternative to mastectomy in women with Paget disease of the breast presenting without evidence of a palpable mass or mammographic density.

\section{REFERENCES}

1. Ashikari R, Park K, Huvos AG, Urban JA. Paget's disease of the breast. Cancer. 1970;26:680-685.

2. Dixon AR, Galea MH, Ellis IO, Elston CW, Blamey RW. Paget's disease of the nipple. Br J Surg. 1991;78:722-723.

3. Chaudary MA, Millis RR, Lane EB, Miller NA. Paget's disease of the nipple: a ten year review including clinical, pathological and immunohistochemical findings. Breast Cancer Res Treat. 1986;8:139-146.

4. Yim JH, Wick MR, Philpott GW, Norton JA, Doherty GM. Underlying pathology in mammary Paget's disease. Ann Surg Oncol. 1997;4:287-292.

5. Nance FC, DeLoach DH, Welsh RA, Becker WF. Paget's disease of the breast. Ann Surg. 1970;171:864-872.

6. Fisher B, Costantino J, Redmond C, et al. Lumpectomy compared with lumpectomy and radiation therapy for the treatment of intraductal breast cancer. N Engl J Med. 1993; 328:1581-1586.

7. Fisher B, Dignam J, Wolmark N, et al. Lumpectomy and radiation therapy for the treatment of intraduactal breast cancer: findings from National Surgical Adjuvant Breast and Bowel Project B-17. J Clin Oncol. 1998;16:441-452.

8. Julien JP, Bijker N, Fentiman IS, et al. Radiotherapy in breast-conserving treatment for ductal carcinoma in situ: first results of the EORTC randomised phase III trial 10853. EORTC Breast Cancer Cooperative Group and EORTC Radiotherapy Group. Lancet. 2000;355:528-533.

9. Paone JF, Baker RR. Pathogenesis and treatment of Paget's disease of the breast. Cancer. 1981;48:825-829.

10. Fourquet A, Campana F, Vielh P, Schlienger P, Jullien D, Vilcoq JR. Paget's disease of the nipple without detectable breast tumor: conservative management with radiation therapy. Int J Radiat Oncol Biol Phys. 1987;13:1463-1465.

11. Stockdale AD, Brierley JD, White WF, Folkes A, Rostom AY. Radiotherapy for Paget's disease of the nipple: a conservative alternative. Lancet. 1989;2:664-666.

12. Bulens P, Vanuytsel L, Rijnders A, van der Schueren E. Breast conserving treatment of Paget's disease. Radiother Oncol. 1990;17:305-309.

13. Kollmorgen DR, Varanasi JS, Edge SB, Carson WE. Paget's disease of the breast: a 33-year experience. J Am Coll Surg. 1998;187:171-177.

14. Pierce LJ, Haffty BG, Solin LJ, et al. The conservative management of Paget's disease of the breast with radiotherapy. Cancer. 1997;80:1065-1072.

15. Fleming ID, Cooper JS, Henson DE, et al., eds. American Joint Committee on Cancer staging manual, 5th ed. Philadelphia: J.B. Lippincott, 1997:171-178.

16. Kaplan EL, Meier P. Nonparametric estimation from incomplete observations. J Am Stat Assoc. 1958;53:457-481.

17. Harris JR, Levene MB, Svensson G, Hellman S. Analysis of cosmetic results following primary radiation therapy for atages I and II carcinoma of the breast. Int J Radiat Oncol Biol Phys. 1979;5:257-261.

18. Lagios MD, Westdahl PR, Marye RR, Concannon S. Paget's disease of the nipple. Cancer. 1984;54:545-551.

19. Dixon AR, Galea RR. Pathogenesis and treatment of Paget's disease of the breast. Cancer. 1981:48:825-829.

20. Anelli A, Anelli TF, McCormick B, Senie R, Borgen PI. Conservative management of Paget's disease of the nipple [abstract]. Am Soc Clin Oncol. 1995;14:100. 
21. Polgar C, Orosz Z, Kovacs T, Fodor J. Breast-conserving therapy for Paget disease of the nipple. Cancer. 2002;94: 1904-1905.

22. Bijker N, Rutgers EJT, Duchateau L, Peterse JL, Julien JP, Cataliotti L. Breast-conserving therapy for Paget disease of the nipple: a prospective European Organization for Research and Treatment of Cancer Study of 61 patients. Cancer. 2001;91:472-477.

23. Silverstein MJ, Lagios MD, Martino S, et al. Outcome after invasive recurrence in patients with ductal carcinoma in situ of the breast. J Clin Oncol. 1998;16:1367-1373.

24. Fowble B, Hanlon AL, Fein DA, et al. Results of conservative surgery and radiation for mammographically detected ductal carcinoma in situ (DCIS). Int J Radiat Oncol Biol Phys. 1997;38:949-957.

25. Solin LJ, Fourquet A, Vicini FA, et al. Mammographically detected ductal carcinoma in situ of the breast treated with breast-conserving surgery and definitive irradiation: long- term outcome and prognostic significance of patient age and margin status. Int J Radiat Oncol Biol Phys. 2001;50:9911002.

26. Bradley SJ, Weaver EW, Bowman DL. Alternatives in the surgical management of in situ breast cancer: a meta-analysis of outcome. Am Surg. 1990;56:428-432.

27. Salvadori B, Fariselli G, Saccozzi R. Analysis of 100 cases of Paget's disease of the breast. Tumori. 1976;62:529-536.

28. Nance FC, DeLoach DH, Welsh RA, Becker WF. Paget's disease of the breast. Ann Surg. 1970;171:864-872.

29. Bijker N, Peterse JL, Duchateau L, et al. Risk factors for recurrence and metastasis after breast-conserving therapy for ductal carcinoma-in-situ: analysis of European Organization for Research and Treatment of Cancer trial 10853. J Clin Oncol. 2001;19:2263-2271.

30. Chan KC, Knox WF, Sinha G, et al. Extent of excision margin width required in breast conserving surgery for ductal carcinoma in situ. Cancer. 2001;91:9-16. 